\title{
Magnetic structure of vector mesons in lattice QCD
}

\author{
E.V. Luschevskaya ${ }^{1,2, a}$, O.E. Solovjeva ${ }^{1}$, O.V. Teryaev ${ }^{4,3}$, and M.S. Prokudin ${ }^{1,5}$ \\ ${ }^{1}$ Institute for Theoretical and Experimental Physics named by A.I.Alikhanov of NRC Kurchatov Institute, \\ 117218, Bolshaya Cheremushkinskaya 25, Moscow, Russia \\ ${ }^{2}$ Moscow Institute of Physics and Technology, Dolgoprudnyj, Institutskij lane 9, Moscow Region 141700, \\ Russia \\ ${ }^{3}$ Lomonosov Moscow State University, GSP-1, Leninskie Gory, 119991 Moscow, Russia \\ ${ }^{4}$ Joint Institute for Nuclear Research, Dubna, 141980, Russia \\ ${ }^{5}$ L.D. Landau Institute for Theoretical Physics, RAS, 142432, Moscow Region, Chernogolovka, Akademika \\ Semenova av., 1-A
}

\begin{abstract}
We have explored the response of the charged vector mesons on the external magnetic field. The $g$-factor of the charged vector $\rho$ and $K^{*}$ mesons has been found. For the charged vector $\rho$ meson the magnetic polarizability and hyperpolarizability have been calculated depending on the spin projection on the the magnetic field. We have estimated the intervals of the magnetic field for which the nonlinear terms in field give a significant contribution to the meson energy.
\end{abstract}

\section{Introduction}

The investigation of the internal structure of mesons in the strong external magnetic field presents a huge interest for the physics of strong interactions. The $g$-factor of vector mesons appears due to their nonzero spin and differs slightly from the value predicted by electrodynamics and theory of gravity for the particles with spin equal one [1,2]. This difference reveals the contribution of strong interactions and its accurate calculation is of interest. The magnetic dipole polarizability and hyperpolarizability are also important physical quantities which characterize the distribution of the fermionic currents inside the meson. They give a significant contribution to the meson energy only at very high magnetic field of hadronic scale, while in a very wide interval of the magnetic fields we observe almost a linear energy dependence on the field. Nonlinear effects appear only in a strong magnetic field.

\section{Details of the calculations}

For the analysis we use the 250-300 lattice $S U(3)$ gauge configurations. They were generated using the tadpole tadpole improved Lüscher-Weisz action at lattice spacings $a=0.086,0.095,0.105,0.115$ fm and lattice volumes $16^{4}, 18^{4}, 20^{4}$.

\footnotetext{
ae-mail: luschevskaya@itep.ru
} 
The fermionic spectrum has been found with the use of Neuberger chirally symmetric overlap operator [3]. The external magnetic Abelian field in the symmetric gauge

$$
A_{\mu}^{B}(x)=\frac{B}{2}\left(x_{1} \delta_{\mu, 2}-x_{2} \delta_{\mu, 1}\right)
$$

was added in the overlap operator. So, the quark is considered in the resulting gauge field which is the sum of nonabelian $S U(3)$ field of gluons and $U(1)$ magnetic field

$$
A_{\mu i j}=A_{\mu i j}^{g l}+A_{\mu}^{B} \delta_{i j}
$$

The magnetic field is quantized in the finite lattice volume [4]

$$
q B=\frac{2 \pi n_{B}}{\left(a N_{s}\right)^{2}}, \quad n_{B} \in \mathbb{Z}
$$

and directed along the ' $z$ ' axis, $a N_{s}$ is the lattice spatial extention in physical units, $q=-1 / 3 e$ is the quark charge. We consider the $n_{B}$ values far from the saturation regime.

Fermionic propagators are constructed as a sum from quark eigenvectors $\psi_{k}$ and eigenvalues $\lambda_{k}$

$$
D^{-1}(x, y)=\sum_{k<M} \frac{\psi_{k}(x) \psi_{k}^{\dagger}(y)}{i \lambda_{k}+m_{q}},
$$

where $x$ and $y$ are the lattice, $M=50$ is the number of Wilson-Dirac eigenmodes used for the propagator approximation, $m_{q}$ is the bare quark mass.

The correlation function of the vector meson is constructed from the propagators of the up- and down-quarks

$$
\langle O(x) \bar{O}(y)\rangle=-\operatorname{Tr}\left[\Gamma_{1} D_{u}^{-1}(x, y) \Gamma_{2} D_{d}^{-1}(y, x)\right],
$$

where $O$ is the meson interpolation operator, $\Gamma_{1}, \Gamma_{2}=\gamma_{\mu}$ are the gamma matrices, $D_{u, d}^{-1}$ are the propagators of $u(s)$ and $d$-quarks. In the expansion of the Euclidean correlation function in the series over the eigenstates of Hamiltonian

$$
C\left(n_{t}\right)=\left\langle\psi^{\dagger}\left(\mathbf{0}, n_{t}\right) \Gamma_{1} \psi\left(\mathbf{0}, n_{t}\right) \psi^{\dagger}(\mathbf{0}, 0) \Gamma_{2} \psi(\mathbf{0}, 0)\right\rangle_{A}=\sum_{k}\langle 0|\hat{O}| k\rangle\left\langle k\left|\hat{O}^{\dagger}\right| 0\right\rangle e^{-n_{t} a E_{k}}
$$

the leading contribution gives the first term with the ground state energy $E_{0}$. Therefore, we can find it from the effective mass plato at large $n_{t}$ values, where $a n_{t}$ is the lattice time in physical units.

The conception of the density matrix enables to extract the energy for the each spin projection on the field axis. To find the energy of the vector meson with $s_{z}=0$ we use the correlation function of the fermionic currents in ' $z$ ' direction

$$
C\left(s_{z}=0\right)=C_{33}
$$

where ' 3 ' corresponds to the $\Gamma_{1}, \Gamma_{2}=\gamma_{3}$ in 5 . The following combinations of the correlation functions

$$
C\left(s_{z}= \pm 1\right)=C_{11}+C_{22} \pm i\left(C_{12}-C_{21}\right) .
$$

give the energies with $s_{z}=+1$ and $s_{z}=-1$ respectively, the notation ' 1 ' and '2' correspond to the $\Gamma_{1}=\gamma_{1}$ and $\Gamma_{2}=\gamma_{2}$ in (5). 


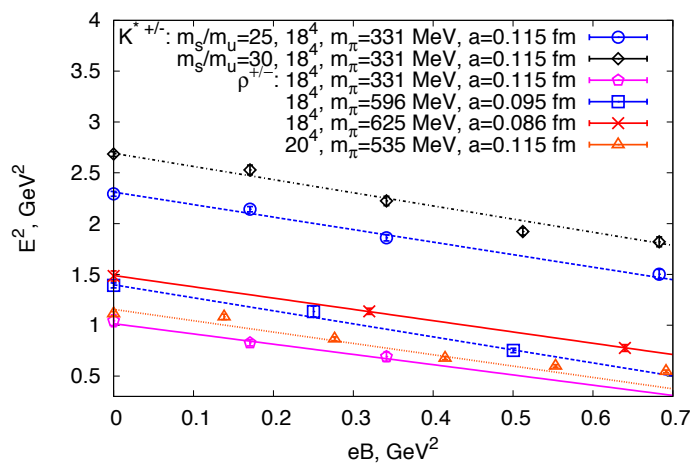

Figure 1. The energy squared of the vector mesons $K^{* \pm}$ and $\rho^{ \pm}$depending on the magnetic field value for various lattice spacings, pion masses, strange quark masses and lattice volumes.

\section{3 g-factor of vector mesons}

At small magnetic field values the meson ground state energy can be described by the linear dependence

$$
E^{2}=|q B|-g s_{z} q B+m^{2},
$$

where $q$ is the particle charge, $s_{z}$ is the spin projection on the axis directed along the external magnetic field $B, g$ is the $g$-factor, $m$ is the mass of the particle or its energy at $B=0$. We neglected the terms with higher power of magnetic field since their contributions is less then $10 \%$.

\begin{tabular}{c|r|r|r|r|r}
\hline$V$ & $m_{\pi}(\mathrm{MeV})$ & $a(\mathrm{fm})$ & $e B\left(\mathrm{GeV}^{2}\right)$ & $g$-factor & $\chi^{2} /$ d.o.f. \\
\hline $18^{4}$ & $331 \pm 7$ & 0.115 & {$[0,0.35]$} & $2.01 \pm 0.18$ & 0.826 \\
\hline $18^{4}$ & $395 \pm 6$ & 0.115 & {$[0,0.35]$} & $2.17 \pm 0.18$ & 0.969 \\
\hline $18^{4}$ & $541 \pm 3$ & 0.115 & {$[0,0.35]$} & $2.12 \pm 0.07$ & 1.159 \\
\hline $18^{4}$ & $667 \pm 3$ & 0.115 & {$[0,0.35]$} & $2.07 \pm 0.19$ & 1.695 \\
\hline $18^{4}$ & $625 \pm 21$ & 0.086 & {$[0,0.70]$} & $2.11 \pm 0.01$ & 0.153 \\
\hline $18^{4}$ & $596 \pm 12$ & 0.095 & {$[0,0.55]$} & $2.30 \pm 0.12$ & 1.094 \\
\hline $18^{4}$ & $572 \pm 16$ & 0.105 & {$[0,0.45]$} & $2.05 \pm 0.03$ & 0.644 \\
\hline $20^{4}$ & $535 \pm 4$ & 0.115 & {$[0,0.45]$} & $2.22 \pm 0.08$ & 1.398 \\
\hline
\end{tabular}

Table 1. The $g$-factor of charged $\rho$ meson and its statistical error are represented. The lattice volumes, the pion masses, the lattice spacings and the interval of the magnetic fields used for the fit are shown in the first to fourth columns respectively.

From Fig. 1 we see that at relatively low field values the energy squared of the $\rho^{+/-}$and $K^{*+/-}$ $\left(s_{z}=+/-1\right)$ is well described by the linear dependence (9) within the errors at $e B \lesssim 0.5 \mathrm{GeV}^{2}$. In Fig. 1 the energy squared of the $\rho$ meson is shown for the different pion masses, two lattice volumes $18^{4}$ and $20^{4}$, various lattice spacings $0.086 \mathrm{fm}, 0.095 \mathrm{fm}$ and $0.115 \mathrm{fm}$. The energy of the vector $K^{*}$ meson is shown for the single pion mass $331 \mathrm{MeV}$, lattice spacing $0.115 \mathrm{fm}$ and lattice volume $18^{4}$ for two ratio values of the bare strange and up-quark masses $m_{s} / m_{u}=25,30$. The $g$-factor was obtained 


\begin{tabular}{c|r|r|r|r}
\hline \hline$m_{\pi}(\mathrm{MeV})$ & $m_{s} / m_{u}$ & $g$-factor & $\chi^{2} /$ d.o.f. & fit, $e B\left(\mathrm{GeV}^{2}\right)$ \\
\hline $331 \pm 7$ & 20 & $2.27 \pm 0.18$ & 1.845 & {$[0,0.35]$} \\
\hline $331 \pm 7$ & 25 & $2.23 \pm 0.23$ & 1.986 & {$[0,0.35]$} \\
\hline $331 \pm 7$ & 30 & $2.29 \pm 0.19$ & 1.366 & {$[0,0.35]$} \\
\hline \hline
\end{tabular}

Table 2. The g-factor of the $K^{* \pm}$ meson, obtained on the lattice with the volume $18^{4}$, the spacing $0.115 \mathrm{fm}$ and various bare masses of the light and strange quarks. The values of $\chi^{2} /$ d.o.f. and intervals of $B$ used for the fitting procedure are also represented.

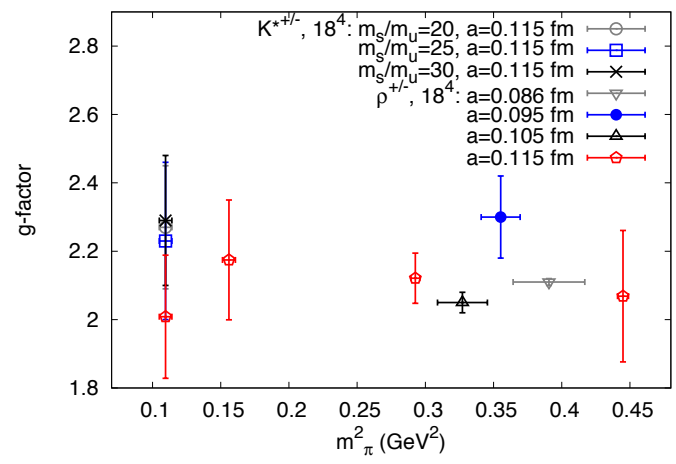

Figure 2. The $g$-factor of the charged vector $\rho$ and $K^{*}$ mesons versus the pion mass squared for the lattice volumes $18^{4}$ and various lattice spacings. For the $K^{*}$ meson the ratio of the bare strange quark mass to the up-quark mass is shown.

as the fit parameter of (9) at $q s_{z}=+1$. Their values are collected for the $\rho$ meson in Table 1 and for the $K^{*}$ meson in Table 2. In Fig. 1 the $g$ value is shown depending on the pion mass squared for the $K^{*}$ and $\rho$ mesons, the errors of the pion mass determination are also depicted. There are no any systematic dependence of the $g$-factor on the lattice volume, the lattice spacing and the pion mass, however the energy are strongly dependent on these parameters. For the $\rho$ meson the relative systematic error is $5 \%$ and the mean value $\bar{g}=2.1$.

Obtained $g_{\rho}$ values are in accordance with the previous results obtained within $S U(3)$ pure lattice gauge theory [5-7]. The magnetic moment of the $\rho$ meson calculated in $2+1$ lattice QCD at different pion masses [6] is higher for approximately $5-10 \%$ than pure gauge result. The analysis of the BaBar experimental data gave the value $g_{\text {exp }}=2.1 \pm 0.5$ [8]. The $g$-factor of the $K^{*}$ meson agrees with the QCD sum rules $2.0 \pm 0.4$ [9] and the other lattice predictons [5, 7].

\section{Magnetic polarizability and hyperpolarizability}

In order to take into account the response of a meson to the magnetic field we added the terms with magnetic polarizability and hyperpolarizability. From the parity conservation it follows that the energy of the charged vector meson can be described by the following formula

$$
E^{2}=|q B|+m^{2}-4 \pi m \beta_{m}(q B)^{2}-4 \pi m \beta_{m}^{h 2}(q B)^{4}-\ldots
$$


for the spin projection $s_{z}=0$ on the external magnetic field and by the dependency

$$
E^{2}=|q B|-g s_{z} q B+m^{2}-4 \pi m \beta_{m}(q B)^{2}-4 \pi m \beta_{m}^{h 1}(q B)^{3}-4 \pi m \beta_{m}^{h 2}(q B)^{4}-\ldots
$$

for the spin projection $s_{z}= \pm 1$, where $\beta_{m}$ is the magnetic dypole polarizability, $\beta_{m}^{h 1}$ and $\beta_{m}^{h 2}$ are the magnetic hypolarizabilties of the first and second order respectively. The calculation of the magnetic polarizability and hyperpolarizabilities is interesting because their experimental determination presents difficulties.

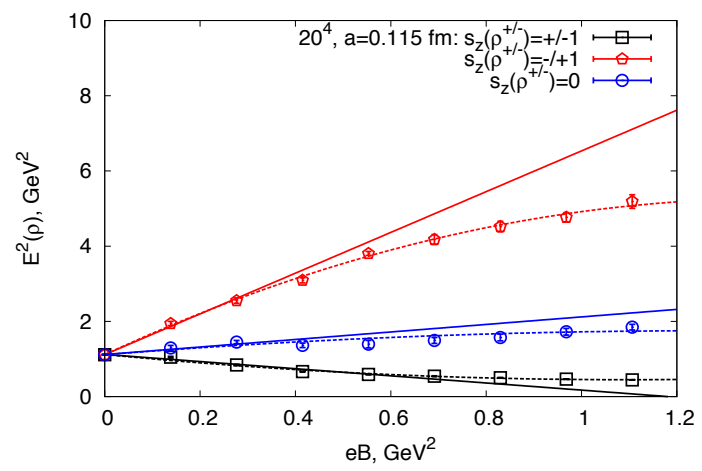

Figure 3. Energy squared dependence on the magnetic field for the lattice volume $20^{4}$, lattice spacing $a=$ $0.115 \mathrm{fm}$ and spin projections $s_{z}=0, \pm 1$.

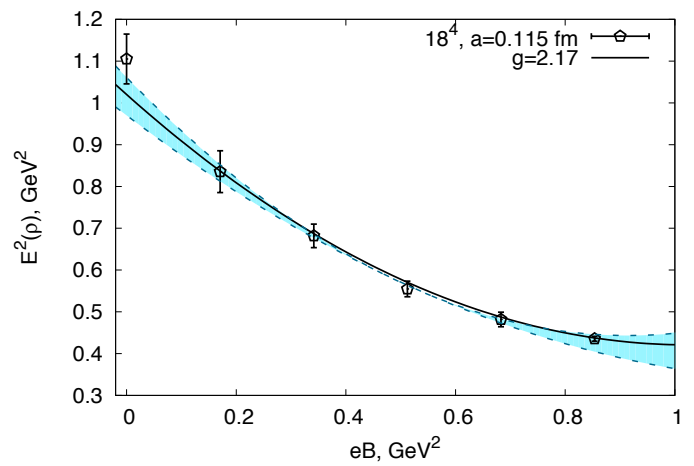

Figure 4. Energy squared dependence on the magnetic field value for the lattice volume $18^{4}$, lattice spacing $0.115 \mathrm{fm}$, pion mass $541 \mathrm{MeV}$ at fixed $g$-factor. The shaded area shows allowable energy values due to the $g$-factor error.

We have found that the terms with hyperpolarizabilities give insignificant contribution to the energy value within the error range at $e B \in[0,1.2] \mathrm{GeV}^{2}$, but at larger fields these terms have to be taken into account. It can be seen from Fig. 3 where the energy squared is shown for the lattice volume $20^{4}$, the lattice spacing $0.115 \mathrm{fm}$, the pion mass $535 \mathrm{MeV}$ and various spin projections $s_{z}=0, \pm 1$ on the field axis. The fits were done using formulas (10) and (11) keeping the terms till second order degree 


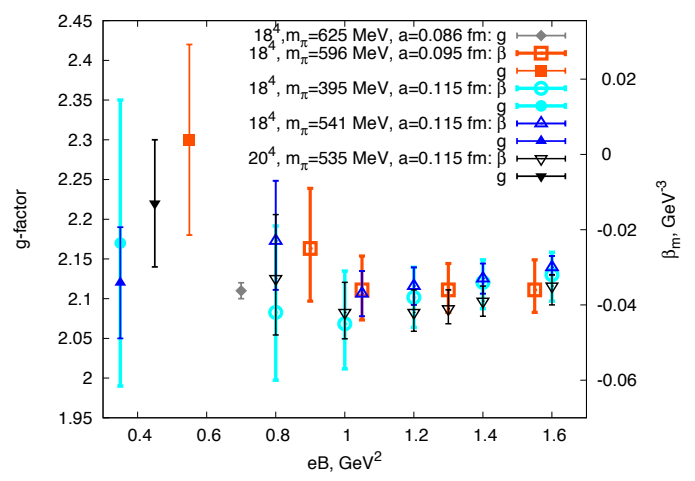

Figure 5. The $g$-factor and the magnetic dypole polarizability $\beta_{m}$ are shown versus the magnetic field value. The data are represented for various lattices and pion masses.

\begin{tabular}{c|r|r|r|r|r}
\hline \hline$V$ & $m_{\pi}(\mathrm{MeV})$ & $a(\mathrm{fm})$ & $\beta_{m}\left(\mathrm{GeV}^{-3}\right)$ & $\chi^{2} /$ d.o.f & fit, $e B\left(\mathrm{GeV}^{2}\right)$ \\
\hline $18^{4}$ & $596 \pm 12$ & 0.095 & $-0.025_{-0.014}^{+0.016}$ & 1.656 & {$[0,0.9]$} \\
\hline $18^{4}$ & $596 \pm 12$ & 0.095 & $-0.036_{-0.006}^{+0.007}$ & 1.864 & {$[0,1.3]$} \\
\hline $18^{4}$ & $541 \pm 3$ & 0.115 & $-0.037_{-0.005}^{+0.006}$ & 2.774 & {$[0,1.05]$} \\
\hline $20^{4}$ & $535 \pm 4$ & 0.115 & $-0.042_{-0.008}^{+0.008}$ & 2.274 & {$[0,1]$} \\
\hline $18^{4}$ & $395 \pm 6$ & 0.115 & $-0.045_{-0.012}^{+0.011}$ & 0.823 & {$[0,1]$} \\
\hline \hline
\end{tabular}

Table 3. The magnetic dipole polarizabilty $\beta_{m}$ of the charged $\rho$ meson for various lattice volumes $V$, lattice spacings $a$ and pion masses. The fit interval is shown in the last column. The results were obtained from the 2-parametric fit at fixed $g$-factor from the low energy sublevel.

in the magnetic field. The fits of the third and the fourth field degree coincide with the presented ones, their contribution is zero within the errors.

To obtain the magnetic dypole polarizability we fit the lattice data by the formula

$$
E^{2}=|q B|-g s_{z} q B+m^{2}-4 \pi m \beta_{m}(q B)^{2},
$$

where $\beta_{m}$ and $m$ are the fit parameters, $q s_{z}=+1$. We use the $g$-factor values from the Table 1. In Fig.4 the energy squared is shown for the lattice volume $18^{4}$, the lattice spacing $a=0.115 \mathrm{fm}$ and the pion mass $m_{\pi}=395(6) \mathrm{MeV}$. The shaded area shows the range of possible energy values when we change the $g$-factor within the error range. The $\beta_{m}$ values obtained in such a way are shown in Table 3 . We have explored the dependence of the $\beta_{m}$ value on the field interval choosen for the fitting procedure to check whether the other terms in $B$ give a contribution to the energy. In Fig. 5 the $g$-factor and $\beta_{m}$ values are represented versus the fit interval. One can see that $\beta_{m}$ begins to increase slowly at $e B>1 \mathrm{GeV}^{2}$. It can be interpreted by a contribution of the term with the positive hyperpolarizability.

We also consider the term of the third power in $B$ and fit the data taking into account $\beta_{m}^{1 h}$, so $m$, $\beta_{m}$ and $\beta_{m}^{h 1}$ are the fit parameters. We used the values of $g$-factor from Table 1. In Fig.6 the solid lines correspond to the boundary $g$-factor values, the values of the $\beta_{m}$ and $\beta_{m}^{1 h}$ are shown in Table 4 with the 


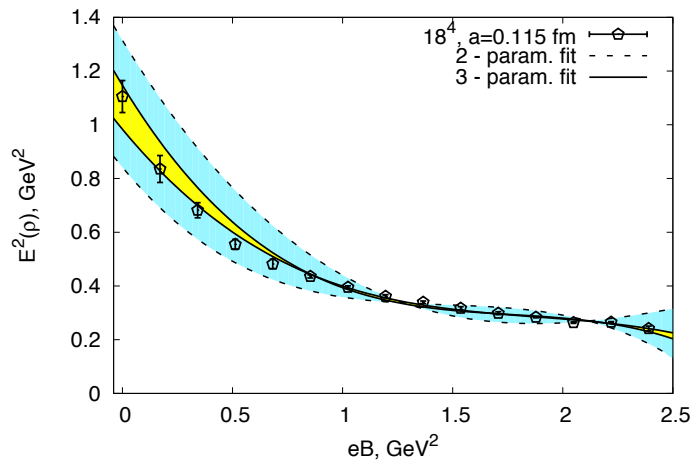

Figure 6. Energy squared dependence on the magnetic field for the lattice volume $18^{4}$, lattice spacing $0.115 \mathrm{fm}$ and pion mass $541 \mathrm{MeV}$. The 2-parametric fit is obtained at fixed $g$ and $\beta_{m}$ values and 3-parametric fit is carried out at fixed $g$-factor.

corresponding lattice parameters, pion masses, the field interval and $\chi^{2} /$ d.o.f. The 2-parametric and 3 -parametric fits give the consistent magnetic dypole polarizabilities, the magnetic hypopolarizability of the first order $\beta_{m}^{h 1}>0$. The dashed lines corresponds to the boundary cases when $g$-factor and $\beta_{m}$ are fixed, $\beta_{m}$ is presented in Table 3. In this case the $\beta_{m}^{h 1}$ is in accordance with the results from Table 4. If we do not fix the $g$-factor and use the 4-parametric fit then the all our conclusions do not change, however the results have worst accuracy. We assume that the hypopolarizabilities of higher orders give a smaller contribution than the first-order one and do not observe the contribution of the fourth-power term in the field. At $e B=0.3-0.4 \mathrm{GeV}^{2}$ the contribution of the term with the

\begin{tabular}{c|r|r|r|r|r|r}
\hline \hline$V$ & $m_{\pi}(\mathrm{MeV})$ & $a(\mathrm{fm})$ & $\beta_{m}\left(\mathrm{GeV}^{-3}\right)$ & $\beta_{m}^{1 h}\left(\mathrm{GeV}^{-5}\right)$ & $\chi^{2} /$ d.o.f & $e B, \mathrm{GeV}^{2}$ \\
\hline $18^{4}$ & $596 \pm 12$ & 0.095 & $-0.050_{-0.008}^{+0.009}$ & $0.009_{-0.003}^{+0.002}$ & 1.965 & {$[0,2.5]$} \\
\hline $18^{4}$ & $541 \pm 3$ & 0.115 & $-0.045_{-0.005}^{+0.005}$ & $0.009_{-0.001}^{+0.001}$ & 2.787 & {$[0,2.5]$} \\
\hline $20^{4}$ & $535 \pm 4$ & 0.115 & $-0.058_{-0.008}^{+0.008}$ & $0.013_{-0.003}^{+0.002}$ & 2.697 & {$[0,2.0]$} \\
\hline $18^{4}$ & $395 \pm 6$ & 0.115 & $-0.047_{-0.009}^{+0.009}$ & $0.009_{-0.002}^{+0.002}$ & 2.255 & {$[0,2.5]$} \\
\hline \hline
\end{tabular}

Table 4. The magnetic dipole polarizabilty $\beta_{m}$, the magnetic hyperpolarizability $\beta_{m}^{1 h}$ of the charged $\rho$ meson obtained from the 3-parametric fit at fixed $g$-factor, where we use the data presented in Table 1. The results are obtained for various lattices and pion masses $m_{\pi}, \chi^{2} /$ d.o.f. values correspond to the fit at the average $g$ value.

dypole magnetic polarizability is not larger than the $g$-factor error. At $e B \sim 1 \mathrm{GeV}^{2}$ the contribution of the hyperpolarizability term is compatible with its error and constitutes $20 \%$. The term with hyperpolarizability $\beta_{m}^{1 h}$ partially compensates the quadratic term in field at not large fields. The lowest energy sublevel of the vector meson does not cross zero at $e B \sim 1 \mathrm{GeV}^{2}$ due to the nonlinear behavior on the magnetic field.

According to the symmetries of the theory the absolute values of the magnetic (hyper)polarizabilities $\beta_{m}^{1 h}$ are equal for the $s_{z}=-1$ and $s_{z}=+1$ cases. The values of the dypole 
magnetic polarizability for the $s_{z}=0$ spin also have been found fitting the lattice data by the formula

$$
E^{2}=|q B|+m^{2}-4 \pi m \beta_{m}(q B)^{2},
$$

at $e B \in[0,1.2] \mathrm{GeV}^{2}$, where $m, \beta_{m}$ are the fit parameters. The fit for the lattice volume equal $20^{4}$ is shown in Fig. 3. The values of $\beta_{m}$ are collected in Table 5 for two different lattice volumes.

\begin{tabular}{c|r|r|r|r}
\hline \hline$V$ & $m_{\pi}(\mathrm{MeV})$ & $a(\mathrm{fm})$ & $\beta_{m}\left(\mathrm{GeV}^{-3}\right)$ & $\chi^{2} /$ d.o.f. \\
\hline $18^{4}$ & $541 \pm 3$ & 0.115 & $0.026 \pm 0.004$ & 1.959 \\
\hline $20^{4}$ & $535 \pm 4$ & 0.115 & $0.026 \pm 0.005$ & 1.365 \\
\hline \hline
\end{tabular}

Table 5. The magnetic dipole polarizability of the charged $\rho$ with $s_{z}=0$ meson obtained for the lattice spacing $0.115 \mathrm{fm}$, the lattice volumes $18^{4}$ and $20^{4}$ from the fit 13 . The pion masses are represented in the second column, the values of $\chi^{2} /$ d.o. $f$ are shown in the last one.

\section{Conclusion}

We have explored the energy behavour of the vector $\rho^{ \pm}$and $K^{* \pm}$ mesons and have calculated the $g$ factor, the magnetic dypole polarizability and hyperpolarizability of the first order. We also have tried to differ the field regimes where the quadratic and other terms in the field contribute significantly to the energy value. The $\beta_{m}$ absolute value and its sign depend on the meson spin projection on the magnetic field axis. The energy of mesons depends on the lattice parameters and the pion mass, however the magnetic characteristics do not within the errors. The observed nonlinear behavior on the magnetic field tends to be a sign changing and prevents the tachyonic mode formation contrary to the prediction of the paper [10].

\section{Acknowledgements}

The authors are grateful to FAIR-ITEP supercomputer center where these numerical calculations were performed. The work of M. Prokudin was completely supported by a Grant from the Russian Science Foundation (grant number RNF-16-12-10151).

\section{References}

[1] O. V. Teryaev, Front. Phys. (Beijing) 11111207 (2016)

[2] H. R. Grigoryan and A. V. Radyushkin, Phys. Lett. B 650421 (2007) [hep-ph/0703069]

[3] H. Neuberger, Phys. Lett. B 417141 (1998)

[4] G.'t Hooft, Nucl. Phys. B 153 (1979) 14

[5] J.N. Hedditch, W. Kamleh, B.G. Lasscock, D.B. Leinweber, A.G. Williams and J.M. Zanotti, Phys. Rev. D 75094504 (2007)

[6] B. Owen et al. Phys. Rev. D 91074503 (2015)

[7] F.X. Lee, S. Moerschbacher and W. Wilcox, Phys. Rev. D 78094502 (2008)

[8] D.G. Gidino and G.T. Sanchez, Int. J. Mod. Phys. A 301550114 (2015)

[9] T.M. Aliev, A. Özpineci and M. Savc1, Phys. Lett. B 678470 (2009)

[10] M.N. Chernodub, Phys. Rev. D 82085011 (2010) 